\title{
Radiological Modalities in the Diagnosis and Screening of Liver Cirrhosis and its Complications
}

\author{
Arshan Dehbozorgi ${ }^{1}$, Pragati Kumar ${ }^{2}$ \\ ${ }^{1}$ University of Missouri School of Medicine, Columbia, Missouri \\ ${ }^{2}$ Department of Radiology, University of Missouri Health Care, Columbia, Missouri \\ Correspondence: Pragati Kumar (kumarp@health.missouri.edu) \\ Received September 7, 2016; accepted December 28, 2016
}

\section{Background}

Liver cirrhosis is the pathologic outcome of many chronic liver diseases, in which repeated injury to the liver results in fibrosis, scarring, and ultimately functional impairment (1). The classic defining histological evaluation of cirrhosis will reveal diffuse regenerative nodules surrounded by dense fibrosis, with parenchymal distortion and collapse causing disruption in hepatic vascular structures (2). There are many causes of cirrhosis, such as viral hepatitis, alcohol related fatty liver disease, non-alcoholic fatty liver disease (NAFLD), biliary diseases, Wilson's disease, alpha1-antitrypsin deficiency, and hemochromatosis $(1,3)$. Cirrhosis is regarded as an end-stage disease that has a high mortality, and liver transplantation serves as the only true curative treatment (2). However, due to recent advances in treatment and management of patients with chronic liver disease, early detection allows for early treatment, which leads to favorable patient outcomes (4). Imaging and image-guided procedures have a role in prevention, screening, diagnosis, and management of cirrhosis (2).

It is important to establish the diagnosis of cirrhosis early in the disease process for a variety of reasons. It has been shown that fibrosis even in later stages of disease may regress with specific therapy, such as in the case with chronic hepatitis B and chronic hepatitis $C(2)$. The challenge is that chronic liver disease is often asymptomatic until the patient develops cirrhosis and decompensates with signs of sepsis, ascites, variceal hemorrhage, encephalopathy, or non-obstructive jaundice (2). The gold standard for diagnosis of cirrhosis has traditionally been a liver biopsy $(3,4)$. Currently, minimally invasive methods such as radiological imaging (ultrasound, computed tomography, and magnetic resonance imaging) and serum markers have become more favorable options in diagnosis, staging, and grading of chronic liver diseases (4). Most physicians rely on imaging and clues of impaired hepatic function as the major basis for diagnosis of cirrhosis. The prognosis is highly variable for cirrhosis with a one-year mortality ranging from $1 \%-57 \%$ depending on the stage (2). 


\section{Ultrasound}

Ultrasound (US) is commonly the first imaging procedure performed during the evaluation of suspected liver disease. The role of ultrasound in cirrhosis includes screening for hepatocellular carcinoma (HCC) and diagnosis of cirrhosis, portal hypertension, and HCC. Ultrasound is readily available, relatively inexpensive, radiationfree, and offers real time evaluation of the liver parenchyma, border, vascular architecture, and vascular flow $(4,5)$. Normal liver parenchyma has a homogenous echotexture with marginally higher echogenicity compared to the adjacent kidney (6). Normal hepatic vessels have smooth walls with anechoic lumens and low resistance waveforms; normal portal veins have thin echogenic walls and monophasic waveforms; and normal hepatic veins lack discernible walls and have a triphasic waveform (6).

Findings of cirrhosis on US include an irregular and nodular surface, coarse echotexture, blunt edges, decreased right lobe-caudate lobe ratio, and indirect evidence of portal hypertension $(1,7)$. Surface nodularity has been shown to be the most common ultrasound feature in detection of cirrhosis (8). The alternating areas of necrosis and regenerative nodules result in areas of parenchymal collapse and bulging. Ultrasound with linear array transducers can provide high-resolution images of the capsule of the liver, picking up even subtle cases of nodularity (9). Although nodularity is a fairly specific sign for cirrhosis, other differential diagnoses include subcapsular metastases, peritoneal seeding, or pseudomyxoma peritonei, which can all mimic cirrhosis (9). Ultrasound can detect cirrhosis with an accuracy of 64-79\%, sensitivity of 52-69\%, and specificity of $74-89 \%$ (1). The ability to additionally evaluate for other signs and complications of cirrhosis, such as dilated portal vein/portosystemic collaterals, splenomegaly and ascites indicating portal hypertension, makes ultrasound an even stronger method for evaluation (6).

Color Doppler, a modality used in US, can show portal vein flow, flow reversal, and collateral flow, which help evaluate for portal hypertension. It has been noted that reversal of flow (hepatofugal flow) and/or collateral flow may be the only findings of cirrhosis in otherwise asymptomatic patients, showing the importance of color doppler in the evaluation of cirrhosis (5). Other findings on color Doppler include enlarged, tortuous hepatic arteries (corkscrew appearance) suggesting increased flow velocity (5). There may also be stasis in the hepatic veins as in the case of thrombosis (6). As cirrhosis progresses, the normal triphasic waveform of the hepatic veins become biphasic and even monophasic because of diminished vascular compliance secondary to fibrosis (6). Because cirrhosis is the most common cause of portal hypertension, the findings of portosystemic collaterals or flow reversal should prompt and support evaluation of the diagnosis of cirrhosis (5). The limitations of ultrasound include operator dependence, and difficulty visualizing the entire liver. The main advantage of ultrasound is that it has a high negative predictive value for cirrhosis (8).

Sonographic elastography is a noninvasive and innovative method assessing liver fibrosis, and thus cirrhosis. There are three techniques available: transient elastography (Fibroscan), acoustic radiation force impulse elastography (ARFI), and ShearWave elastography (SWE) (1, 10). For moderate fibrosis (fibrosis stages 1 and 2), the technique of choice is Fibroscan as it produces significantly better results than ARFI elastography (1). The limitations of Fibroscan include missed diagnoses in $2-11 \%$ of 
cases, the operator-dependent nature of the test, difficult measurement in obese patients and ascitic patients and the small volume of liver parenchyma that is measured (1). A meta-analysis by Talwalker et al. showed that for patients with stage IV fibrosis (or cirrhosis), with regards to ultrasound transient elastography (Fibroscan), the pooled sensitivity was $87 \%$ and specificity was $91 \%$, and among stage II-IV fibrosis the sensitivity was $70 \%$ and specificity was $84 \%$ for detection of cirrhosis (11).

\section{Computed tomography}

Abdominal computed tomography (CT) is a frequently used study obtained because of its wide range of utility. As a part of routine evaluation of the CT images, the liver is screened for fibrosis and parenchymal changes. Findings of cirrhosis on CT include an irregular/nodular surface, blunt liver edge, parenchymal and morphological changes, and signs of portal hypertension. The diagnostic accuracy, sensitivity, and specificity have been shown to be $67-86 \%, 77-84 \%$ and $53-68 \%(1,7)$. Using a cirrhosis scoring criteria developed by Harbin et al., with the ratio of transverse caudate lobe width to transverse right lobe width, cirrhotic livers could be separated from non-cirrhotic livers with an accuracy, sensitivity, and specificity of $94 \%, 84 \%$, and $100 \%$ (12). Limitations of diagnostic $\mathrm{CT}$ include cost and radiation exposure, making the radiation free and inexpensive ultrasound more appealing as an initial test. The advantage of CT imaging over ultrasound is its ability to detect $\mathrm{HCC}$ and perform staging post contrast injection (1). The best predictive signs of liver cirrhosis on CT and MRI were liver parenchymal abnormalities, manifestation of portal hypertension, and morphological changes (7). In a

multicenter study review by Kudo et al., MRI and CT were deemed superior in sensitivity to US in predicting cirrhosis since they evaluate the hepatic parenchyma and morphology better than ultrasound (7).

\section{Magnetic resonance imaging}

Magnetic resonance imaging (MRI) is another modality that can detect features of cirrhosis, including surface irregularity, heterogenous enhancement, caudate lobe enlargement, splenomegaly, decreased right to left lobe volume ratio, varices, expanding gallbladder fossa, and ascites (1). The sensitivity and specificity of contrast enhanced MRI for cirrhosis is similar to that of CT. However, by measuring the diameter of hepatic veins, particularly for a right hepatic vein $<7 \mathrm{~mm}$, Zhang et al. shows an increase in detecting cirrhosis with a sensitivity and specificity of $88 \%$ and $85 \%$ (13).

Through the use of MR elastography, double contrast enhanced MRI, diffusionweighted MRI, susceptibility-weighted MRI, and T1rho, the detection of liver fibrosis in pre-cirrhotic stage was detected with great accuracy. MR elastography (MRE) may be the most reliable of these newer MRI based methods, as it appears to be more accurate for detecting fibrosis early and measuring the stiffness of the liver as a whole, with a sensitivity and specificity of $98 \%$ and $99 \%$ (14). Some argue this newer technique may even replace the gold standard biopsy as it allows for evaluation of the entire hepatic volume in an accurate manner. The limitations of MRE are the cost and limitations in patients with hepatitis and congestive heart failure (14). The imaging modalities described above are summarized in table 1. 


\begin{tabular}{|c|c|c|c|}
\hline \multicolumn{4}{|c|}{ Table 1. Imaging modalities in detection of cirrhosis } \\
\hline & Ultrasound & CT & MRI \\
\hline Findings of cirrhosis & $\begin{array}{ll}\text { - } & \text { Surface } \\
\text { irregularity } \\
\text { - } & \text { Coarse } \\
\text { echotexture } \\
\text { - } & \text { Decreased R lobe } \\
\text { - caudate lobe } \\
\text { ratio } \\
\text { - Evidence of } \\
\text { portal } \\
\text { hypertension }\end{array}$ & $\begin{array}{ll}\text { - } & \text { Surface } \\
\text { irregularity } \\
\text { - } & \text { Parenchymal } \\
\text { changes } \\
\text { - } & \begin{array}{l}\text { Signs of portal } \\
\text { hypertension }\end{array} \\
\text { - } & \end{array}$ & $\begin{array}{ll}\text { - } & \text { Surface } \\
\text { irregularity } \\
\text { - } & \text { Heterogenous } \\
\text { enhancement } \\
\text { - } & \text { Caudate lobe } \\
\text { enlargement } \\
\text { - } \\
\text { Decreased R to } \\
\text { L lobe volume } \\
\text { ratio } \\
\text { - Expanding } \\
\text { gallbladder } \\
\text { fossa } \\
\text { - Varices and } \\
\text { Ascites }\end{array}$ \\
\hline Sensitivity/Specificity & $52-69 \% / 74-89 \%$ & $77-84 \% / 53-68 \%$ & $88 \% / 85 \%$ \\
\hline $\begin{array}{l}\text { Role in cirrhosis and } \\
\text { HCC }\end{array}$ & $\begin{array}{ll}\text { - } & \begin{array}{l}\text { Screening and } \\
\text { surveillance }\end{array} \\
\end{array}$ & - Diagnosis & - Diagnosis \\
\hline Pros & $\begin{array}{ll}\text { - } & \text { No radiation } \\
\text { exposure } \\
\text { - } & \text { Low cost and } \\
\text { availability } \\
\text { - } \\
\text { Color doppler } \\
\text { assessment of } \\
\text { flow } \\
\text { - Sonographic } \\
\text { elastography } \\
\text { assessment of } \\
\text { fibrosis } \\
\text { - Highest } \\
\text { specificity }\end{array}$ & $\begin{array}{l}\text { - Higher } \\
\text { sensitivity } \\
\text { than US } \\
\text { - } \begin{array}{l}\text { Visualization } \\
\text { of entire liver }\end{array}\end{array}$ & $\begin{array}{ll}\text { - } & \text { No radiation } \\
\text { exposure } \\
\text { - } & \text { Most sensitive } \\
\text { in detection of } \\
\text { cirrhosis } \\
\text { - } & \text { Good } \\
\text { specificity } \\
\text { - } & \text { Visualize entire } \\
\text { liver } \\
\text { - } \\
\text { Detection of } \\
\text { small HCC }\end{array}$ \\
\hline Cons & 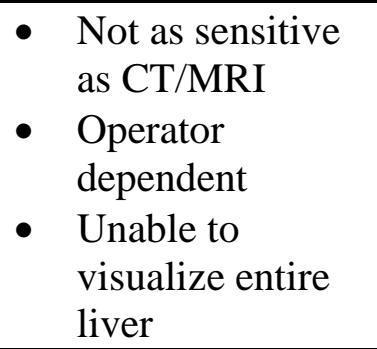 & $\begin{array}{ll}- & \text { Radiation } \\
\text { exposure } \\
\text { - } & \text { Cost is higher } \\
\text { than US }\end{array}$ & $\begin{array}{ll}\text { - } & \text { Cost is higher } \\
\text { than US and CT } \\
\text { - } \\
\text { More time } \\
\text { intensive }\end{array}$ \\
\hline
\end{tabular}




\section{Screening for HCC}

The late presentation of chronic liver disease emphasizes the importance for population screening, especially in high-risk patients. Currently, screening for chronic hepatitis $\mathrm{C}$ is an accepted screening method for patients born between 1945-1965. Other screening methods include NAFLD fibrosis scores for non-alcoholic fatty liver disease and alcohol misuse screening (2). Once diagnosed with cirrhosis, The American Association for the Study of Liver Diseases (AASLD) recommends that patients should be evaluated for esophageal and gastric varices with a screening esophagogastroduodenoscopy (EGD) and treated accordingly to the findings $(15,16)$.

Hepatocellular carcinoma accounts for a large majority of all primary liver cancers, with the highest incidence rates in regions with high hepatitis $\mathrm{B}$ virus and hepatitis $\mathrm{C}$ virus infection (17). Aside from $\mathrm{HBV}$ and $\mathrm{HCV}$, other risk factors for $\mathrm{HCC}$ include alcoholic liver disease, nonalcoholic fatty liver disease, and in general, diseases leading to cirrhosis (17).

In patients with cirrhosis, $\mathrm{HCC}$ has been shown to develop at a rate of $1 \%-4 \%$ per year (18). The American Association for the Study of Liver Diseases (AASLD) has developed a system for surveillance based on the level of risk of HCC (19). For high-risk patients, AASLD recommends ultrasound screening at diagnosis and every six months (19). The screening interval does not need to be shortened for patients at higher risk of HCC (19). The AASLD recommends that HCC surveillance is deemed cost effective if the risk of HCC exceeds $1.5 \%$ per year in patients with hepatitis $\mathrm{C}$ and $0.2 \%$ per year in patients with hepatitis B. They also recommend that alpha-fetoprotein alone lacks the necessary sensitivity and specificity to be effective for surveillance and diagnosis, and although AFP with ultrasound increases detection rates, it also increases false positives and cost (19). In conclusion, ultrasound every six months is the best modality for surveillance.

In the event that a nodule larger than $1 \mathrm{~cm}$ is found on ultrasound screening, the AASLD recommends evaluation with dynamic contrast enhanced MRI or 4-phase multidetector CT (17). If the nodule is smaller than one centimeter, then the patient should have a follow up ultrasound at 3-6 month interval (19). After two years of documented no growth, the patient may return to routine surveillance with a screening ultrasound every six months (19). The AASLD guidelines on surveillance of HCC are summarized in table 2 . 


\begin{tabular}{|c|c|}
\hline \multicolumn{2}{|c|}{ Table 2. Summary of AASLD guidelines on surveillance of HCC } \\
\hline High risk patients & Recommendations \\
\hline $\begin{array}{l}\text {-Hepatitis B } \\
\text { - Asian male hepatitis B carrier with } \\
\text { age }>40 \\
\text { - Asian female hepatitis B carrier } \\
\text { with age }>50 \\
\text { - Hepatitis B carrier with family } \\
\text { history of HCC } \\
\text { - African/North American Blacks } \\
\text { with hepatitis B } \\
\text { - Chronic Hepatitis B carriers } \\
\text {-Hepatitis C cirrhosis } \\
\text {-Stage } 4 \text { primary biliary cirrhosis } \\
\text {-Genetic hemochromatosis and cirrhosis } \\
\text {-Alpha 1-Antitriypsin deficiency and } \\
\text { cirrhosis } \\
\text {-Other cirrhosis }\end{array}$ & $\begin{array}{l}\text { 1. Patients should be entered into } \\
\text { surveillance programs } \\
\text { 2. Surveillance should include } \\
\text { ultrasound at six month intervals }\end{array}$ \\
\hline -Patients on the transplant waiting list & $\begin{array}{l}\text { 1. Screen for } \mathrm{HCC} \text { as it affects priority } \\
\text { for OLT }\end{array}$ \\
\hline $\begin{array}{l}\text { For additional details, refer to: } \\
\text { Bruix, J., \& Sherman, M. (2011). Managen } \\
\text { update. Hepatology, 53(3), 1020-1022 }\end{array}$ & f hepatocellular carcinoma: an \\
\hline
\end{tabular}

\section{Conclusion}

Cirrhosis is an increasing cause of morbidity and mortality that requires accurate and early detection for optimal treatment and management. Ultrasound is commonly the first step in radiological examination in patients suspected of having liver disease. The ultrasound findings in conjunction with using color Doppler to assess for flow velocities allows for better detection of cirrhosis, portal hypertension, and hepatocellular carcinoma. However, nonspecific findings should be further evaluated by CT, MRI, or biopsy depending on the clinical context. Hepatocellular carcinoma accounts for the majority of all primary liver cancers, and develops at an increased rate in patients with cirrhosis. Due to this increased risk, the AASLD has developed guidelines for surveillance and management of patients at risk for HCC. 


\section{References}

1) Huber, A., Ebner, L., Heverhagen, J. T., \& Christe, A. (2015). State-of-the-art imaging of liver fibrosis and cirrhosis: A comprehensive review of current applications and future perspectives. European journal of radiology open, 2, 90-100.

2) Tsochatzis, E. A., Bosch, J., \& Burroughs, A. K. (2014). Liver cirrhosis. The Lancet, 383(9930), 1749-1761.

3) Schuppan, D., \& Afdhal, N. H. (2008). Liver cirrhosis. The Lancet, 371(9615), 838851.

4) Elbagir, S, Mohamed, Y, \& Bushra, A. (2014). Role of CT Scan and Ultrasound Imaging in Characterization of Common Liver Diseases. IJAR Indian Journal of Applied Research.

5) Tchelepi, H., Ralls, P. W., Radin, R., \& Grant, E. (2002). Sonography of diffuse liver disease. Journal of Ultrasound in Medicine, 21(9), 1023-1032.

6) Heller, M. T., \& Tublin, M. E. (2014). The role of ultrasonography in the evaluation of diffuse liver disease. Radiologic Clinics of North America, 52(6), 1163-117.

7) Kudo, M., Zheng, R. Q., Kim, S. R., Okabe, Y., Osaki, Y., Iijima, H., ... \& Usuki, N. (2008). Diagnostic accuracy of imaging for liver cirrhosis compared to histologically proven liver cirrhosis. Intervirology, 51(Suppl. 1), 17-26.

8) Choong, C. C., Venkatesh, S. K., \& Siew, E. P. (2012). Accuracy of routine clinical ultrasound for staging of liver fibrosis. Journal of clinical imaging science, 2(1), 58.

9) Kreuer, S., Elgethun, M., \& Tommack, M. Imaging Findings of Cirrhosis.

10) Berzigotti, A., \& Castera, L. (2013). Update on ultrasound imaging of liver

fibrosis. Journal of hepatology, 59(1), 180-182.

11) Talwalkar, J. A., Kurtz, D. M., Schoenleber, S. J., West, C. P., \& Montori, V. M. (2007). Ultrasound-based transient elastography for the detection of hepatic fibrosis: systematic review and meta-analysis. Clinical gastroenterology and hepatology, 5(10), 1214-1220.

12) Harbin, W. P., Robert, N. J., \& Ferrucci Jr, J. T. (1980). Diagnosis of cirrhosis based on regional changes in hepatic morphology: a radiological and pathological analysis. Radiology, 135(2), 273-283.

13) Zhang, Y., Zhang, X. M., Prowda, J. C., Zhang, H. L., Sant'Anna Henry, C., Shih, G., ... \& Prince, M. R. (2009). Changes in hepatic venous morphology with cirrhosis on MRI. Journal of Magnetic Resonance Imaging, 29(5), 1085-1092.

14) Yin, M., Talwalkar, J. A., Glaser, K. J., Manduca, A., Grimm, R. C., Rossman, P. J., ... \& Ehman, R. L. (2007). Assessment of hepatic fibrosis with magnetic resonance elastography. Clinical Gastroenterology and Hepatology, 5(10), 1207-1213.

15) Garcia-Tsao, G., Sanyal, A. J., Grace, N. D., \& Carey, W. (2007). Prevention and management of gastroesophageal varices and variceal hemorrhage in cirrhosis. Hepatology, 46(3), 922-938.

16) El-Serag, H. B., \& Davila, J. A. (2011). Surveillance for hepatocellular carcinoma: in whom and how?. Therapeutic advances in gastroenterology,4(1), 5.

17) El-Serag, H. B., \& Davila, J. A. (2011). Surveillance for hepatocellular carcinoma: in whom and how?. Therapeutic advances in gastroenterology,4(1), 5.

18) Hussain, S. M., Reinhold, C., \& Mitchell, D. G. (2009). Cirrhosis and Lesion

Characterization at MR Imaging 1. Radiographics, 29(6), 1637-1652. 
19) Bruix, J., \& Sherman, M. (2011). Management of hepatocellular carcinoma: an update. Hepatology, 53(3), 1020-1022. 